\title{
La trop fragile utopie de L'Astrée : une variation du pot de terre contre le pot de fer
}

Certains genres littéraires entretiennent d'étroites relations avec l'utopie : ainsi la pastorale qui, depuis l'Antiquité, se fait prétexte à la description de sociétés idéalisées vivant au sein d'une nature généreuse qui ne demande pas énormément de travail pour récolter beaucoup de fruits ${ }^{1}$. La mode est à nouveau à la bergerie dans l'Europe de la Renaissance : un regain d'intérêt qui accompagne les développements de l'humanisme et offre un temps de pause salutaire à des hommes qui subissent les guerres fratricides de l'époque. C'est en 1607 que commence la parution de L'Astrée d'Honoré d'Urfé : dernier roman pastoral à connaître un succès durable en France, il influence toute une société galante ${ }^{2}$. Si l'on en connaît alors par cœur des passages entiers, aucun auteur ne reprend dans les mêmes dimensions cet idéal pastoral. L'utopie des bergers n'est plus un sujet d'écriture aussi sérieux qu'il a pu l'être : Charles Sorel donne le coup de grâce au genre avec son Berger extravagant en $1627^{3}$. Cet arrêt du genre et le succès que connaît en parallèle l'œuvre d'Urfé a de quoi interroger. Nous verrons en quoi cet auteur reprend, comme Sorel après lui quoique dans un registre comique,

- Pauline Philipps - professeure agrégée, doctorante au CÉRÉdI de l'Université de Rouen Normandie. Adresse de correspondance : CÉRÉdI, Université de Rouen UFR Lettres et Sciences Humaines Rue Lavoisier 76821 Mont-Saint-Aignan Cedex ; e-mail : philipps.pauline@hotmail.fr

ORCID iD : https://orcid.org/0000-0003-0797-1574

1. Marie-Madeleine Fragonard rappelle son rôle de "divertissement " au sens pascalien, en tant qu' "image utopique d'un passé de patriarches ou d'adolescents heureux hors de tout ce qui dégrade la vie réelle en cette période difficile [le XVI ${ }^{e}$ siècle] » (Fragonard, 2000, p. 141).

2. La société galante qui se réclame de L'Astrée cherche à se distinguer d'une galanterie légère et qui ne traite pas de sujets très sérieux ni très renseignés, ce que rappelle Emmanuel Bury, citant une lettre de Huet à Madeleine de Scudéry. "L'érudition » de L'Astrée la met " au-dessus des romans vulgaires, uniquement renfermés dans les bornes de la galanterie» (Bury, 2007, p. 316).

3. Il s'agit d'une entreprise de critique sous des dehors de fiction, comme l'affirme le sous-titre, qui parle des « impertinences des Romans \& de la Poësie ». Pour plus de précisions, voir Rosellini (2015). 
les codes de l'utopie pastorale non pour la défendre, mais pour la remettre en question et la détruire ${ }^{4}$. Avec L'Astrée, le ver est dans le fruit, ou pour reprendre la formule de Genette « le serpent dans la bergerie » (1966, p. 109-122) : en proposant à ses lecteurs de localiser précisément sa société utopique de bergers, Urfé la met en contact direct avec le monde historique et brutal des hommes. La noirceur du temps historique y gangrène progressivement l'utopie dans laquelle évoluaient au départ les personnages principaux. Les contacts avec l'extérieur se font plus violents, jusqu'à annoncer la perte de la région tout entière à la fin de la troisième partie ${ }^{5}$. Nous étudierons dans un premier temps la façon dont Honoré d'Urfé construit son univers forézien comme une utopie qui, bien que localisée sur la carte de France, a des allures de sanctuaire, et nous montrerons que très tôt il devient évident que ces apparences de sérénité ne sont qu'un leurre : les zones de friction avec le monde extérieur attaquent la perfection utopique et la mettent à mal. Ceci nous amènera enfin à nous interroger sur l'invasion progressive de l'utopie mise en scène dans ce roman. L'Astrée ne nous raconte alors rien d'autre que l'histoire du pot de terre contre le pot de fer : quand l'utopie pastorale pensait proposer une solution au dur monde des hommes, l'injustice et la violence de celui-ci finissent par avoir raison d'elle. En s'approchant trop des réalités du temps historique, la pastorale court à sa propre perte : l'auteur ne se mêle d'utopie que pour montrer la fragilité des rêves qu'elle porte. Urfé nous prévient dès son adresse au personnage de Céladon, qui incarne ses idéaux. Proposée en tête de la deuxième partie, celle-ci marque un infléchissement depuis l'adresse qui ornait les débuts de la première partie, et où Astrée était encore présentée comme pouvant vivre au milieu de l'humanité. L'auteur dévoile son vrai rapport à l'utopie à partir de cette deuxième partie.

\section{Un « lieu de nulle part » précisément localisé}

Honoré d'Urfé installe son utopie dans sa région, le Forez ; à cela s'ajoutent des précisions d'ordre historique, puisque les événements racontés sont censés se dérouler aux alentours du Ve siècle. Mais si les personnages évoluent dans un univers dont le nom rappelle une réalité géographique, l'auteur idéalise les lieux. Il ne s'agit pas pour lui de faire dans un réalisme exigeant quand il décrit les bords du Lignon et la société qui s'y est installée, même si l'on note déjà un premier écart avec la tradition arcadienne (Genette, 1966).

4. Jean-Pierre van Elslande a montré le lien qui existait entre cette veine du roman pastoral et la crise des valeurs que traverse alors la France. Voir Elslande (2002).

5. Son continuateur principal, Baro, imagine qu'au prix d'une lutte acharnée le Forez s'en sort pour cette fois. Mais le traumatisme de la crise demeure, et crée un dangereux précédent. 


\section{1. Une vie facile et vertueuse}

La vie parait agréable dans cet endroit préservé de la perversité des hommes : les bergers ne connaissent pas les luttes de pouvoir, et la vie y paraît facile. Elle s'y écoule, semble-t-il, dans un perpétuel printemps, puisque l'on ne voit pas se succéder les saisons. Là où les bergers se montrent les plus actifs, c'est à l'occasion de jeux organisés auxquels on fait lointainement référence, comme la course ${ }^{6}$, et le peu de valeur marchande des prix remportés montre que l'argent même ne représente pas un souci dans cette société idéale. De plus, Urfé présente d'emblée ses personnages principaux comme idéaux. L'adresse à la bergère Astrée qu'il place au début de son roman nous montre un père inquiet de ce que sa fille, pure et candide, veuille voir le monde, peuplé de «monstres horribles [qui] y vont attendants les passants pour les devorer " (Urfé, 2011, I, p. 109). Le lecteur même n'est pas censé pouvoir apprécier tant de perfections, puisque s'adressant à Céladon, Urfé le met en garde en lui expliquant "que l'aage où nous sommes est bien contraire à [son] opinion ", et que l'on se moquera de lui'. L'utopie ainsi présentée dans ce roman semble fonctionner complètement hors-sol, loin des atteintes du monde et de ses perversions. La proximité géographique avec d'autres sociétés, présentées sous un jour volontairement plus réaliste ${ }^{8}$, parait alors n'être voulue que pour mieux faire ressortir les perfections d'un monde idéal, et dénoncer les travers du monde.

\section{2. Un abri pour les malheureux}

Les communications qui s'établissent avec le dehors se font en effet dans un premier temps à l'avantage du Forez : celui-ci devient un abri pour tous les oppressés des tyrannies humaines. Peu de personnages quittent de bon cœur cette région utopique : les seuls à le faire sont les chevaliers de la cour des nymphes, mais alors ils remplissent une mission d'État, et ce n'est pas sans avoir pour dessein de revenir. Ces personnages tiennent en fait d'un univers au sens propre bâtard (Matzat, 2002) : si le Forez a tout d'une utopie, il se scinde en deux parties bien distinctes, et qui ne se rencontrent que rarement. La perfection absolue ne se retrouve que dans la société des bergers, et la cour de la reine Amasis reproduit davantage un modèle bien connu des lecteurs, puisque l'on y retrouve le fonctionnement habituel ou presque d'un royaume. Ce mélange menace déjà l'intégrité de l'utopie, bien que dans la première partie les personnages qui peuplent cette cour semblent ne pas différer grandement des simples ber-

6. On ne nous parle qu'indirectement de ces pratiques, puisqu'une nymphe, Silvie, se contente de rappeler à Céladon que c’était lui qui avait remporté la « course » et gagné pour cela un « chapeau de fleurs » (Urfé, 2011, livre II, p. 171).

7. Il s'agit là d'une posture, et Urfé n'est pas sans savoir que son personnage plaît à son public, puisque nous sommes ici au début de la deuxième partie, et que la première, qui a été publiée trois ans auparavant, a déjà connu un grand succès (Urfé, 2016, p. 27).

8. Pour prouver le paradoxe qu'il y a entre réalisme et fiction dans L'Astrée, Wolfgang Matzat fait référence à la structure de la cour des Nymphes; nous verrons que celle-ci n'est qu'un lieu de transition vers les univers plus réalistes encore des récits secondaires. Voir Matzat (2002). 
gers. Le seul à quitter le mode de vie pastoral pour tenter sa chance dans les sociétés humaines historiques est Alcippe, père de Céladon, qui renonce bien vite à porter l'épée pour garder la houlette. L'utopie ainsi défendue par Urfé constitue une critique des biens du monde contre des joies présentées comme innocentes et pures. La génération qui constitue le vivier principal des personnages foréziens est une génération qui ne connaît pas l'extérieur : l'utopie s'est dans ce sens refermée sur ellemême. Les déplacements ne peuvent se faire alors que dans le sens inverse : le roman se nourrit des récits faits par des étrangers poursuivis par l'injustice humaine et qui trouvent dans ces lieux un sanctuaire. La localisation de cette utopie constitue par conséquent en apparence une vraie force pour elle, rendant possible l'arrivée de nouveaux colons, conscients des différences qui existent entre la dure réalité et le rêve éveillé que l’on y vit.

A priori, préciser l'emplacement de l'utopie et la placer au sein de la Gaule lui est très profitable : les récits que l'on ne cesse de faire de ce qui se passe hors de ses frontières aident alors le projet utopique, peut-être plus encore que la relation précise de ce qui s'y passe, puisque plus le roman avance, plus ces récits prennent de la place, jusqu'à supplanter le récit principal. Sur les bords du Lignon, tout le monde ne fait presque plus qu'écouter les tristes histoires d'un nouveau venu.

\section{Une utopie rongée de l'intérieur par un ver}

\section{1. Une utopie fragilisée par le voisinage de la cour des nymphes}

L'utopie pastorale présentée montre quelques faiblesses, qui annoncent sa chute prochaine. Les premières réticences apparaissent à propos de cette "zone de friction " dont nous avons brièvement parlé : la cour d'Amasis. La continuité dans la perfection entre cet espace et la société des bergers n'est qu'apparente et, avant même que la crise ne s'annonce, on remarque quelques signes qui ne trompent pas. On a vu que ce qui faisait une partie de la force de l'idéal présenté était l'absence de hiérarchie entre les bergers, si ce n'est une hiérarchie héritée de l'idée que les pères avaient une certaine autorité sur leurs enfants9. Or, ces écarts sociaux se retrouvent dans cette cour des nymphes : dès le départ, Galathée, fille de la reine, fait preuve d'une autorité qui, bien que charmante, marque nettement sa conscience de ne pas être du même rang que ses compagnes. Très vite, Céladon, prisonnier de la nymphe, compare sa situation à celle d'un homme retenu dans une prison dorée (Urfé, 2011, livre IV, p. 250). À ceci s'ajoute le fait que les hommes de cette cour sont des chevaliers : qui dit « chevalerie » dit « violence potentielle», puisqu'ils doivent prouver leur valeur et faire la guerre.

9. Le père est alors déjà une sorte de " magistrat domestique " pour reprendre les termes d'Alain Desrayaud. Locke a beau essayer de défendre l'idée d'un rapport plus égalitaire entre pères et enfants, ses théories ont quelque chose de trop novateurs pour être adoptées. Voir Desrayaud (2012). 
Même s'il s'agit d'une violence épique et qui, par conséquent, est mise en valeur ${ }^{10}$, elle représente un danger potentiel. Certes, les principaux chevaliers mentionnés dans cette cour sont tous pleins de qualité : mais ce sont eux qui, déjà, sont à l'origine des fêlures visibles dès le début du roman.

\section{2. Une utopie déjà amputée au début du roman}

Si cet univers pastoral est aussi utopique, c'est parce qu'à l'absence de jalousies liées aux richesses et au pouvoir s'ajoute la certitude que l'on peut avoir dans les sentiments des uns et des autres. Urfé fait de cette région l'endroit où se trouve la «fontaine de vérité d'amour », celle qui révèle à qui s'y mire les sentiments de qui l'intéresse. Dans l'univers pastoral traditionnel, il ne reste plus aux bergers qu'à s'occuper d'amour ; seule la passion amoureuse peut encore venir troubler la paix utopique qui autrement y règne. En imaginant cette fontaine, Urfé effaçait ce dernier obstacle à la création d'un lieu parfait. Or, celle-ci est inaccessible pour les personnages dès l'incipit, ce qui est la cause directe du suicide raté de Céladon, puisque celui-ci se jette à l'eau à cause de la jalousie de sa maîtresse, laquelle a été persuadée par un traitre qu'il en aime une autre. Le responsable de cette tragédie n'est autre que le premier des chevaliers de la cour d'Amasis, son fils, Clidaman. Jaloux, celui-ci avait attiré cette malédiction sur la fontaine. Il a donc, avant même le commencement du roman, détruit ce qui faisait une partie non-négligeable de la perfection de l'utopie forézienne. Chevalier, c'est lui qui jette une malédiction sur une fontaine qui paraissait sortie d'un roman de chevalerie (Gouédo-Thomas, 1992).

\section{3. Le début de nouvelles interactions entre la cour et les bergers}

Si les deux mondes qui se côtoyaient dans cette utopie se mêlent dangereusement, ce n'est qu'à partir d'un incident précis. Auparavant, si parfois leurs habitants se rencontraient, leur différence de statuts interdisait implicitement tout échange réel. Céladon ne reconnaît pas Silvie, et Léonide s'étonne de n'avoir jusqu'ici jamais vraiment parlé aux bergères. Le roman est principalement construit autour de cet incident qui provoque le mélange de ces deux univers : la rencontre que Galathée et ses suivantes font avec Céladon conduit à l'accroissement des échanges. Galathée, sœur de Clidaman, rencontre Céladon. Aussitôt amoureuse de lui, elle le garde chez elle. Léonide, également touchée par ses charmes, finit pour cette raison par devenir familière avec les bergers du Forez. En faisant de ces deux événements les premiers incidents majeurs de son roman, Urfé place son récit principal au moment du choc responsable de la crise que connaît son utopie, plutôt que de nous présenter ce qui fait tout d'abord de sa société pastorale une société utopique. On ne découvre le fonctionnement de celle-ci que petit à petit, et alors principalement sur le mode de la remé-

10. Le grand modèle du genre, L'Iliade, multiplie les formes de combat, et la chanson de geste à sa suite donne à voir en détail les blessures reçues lors de batailles acharnées. Voir par exemple Bonansea (2014). 
moration (Chamard-Bergeron, 2013) : l'utopie que l'on présente n'est donc plus qu'un monde qui appartient au passé. Ce faisant, Urfé choisit le genre du roman : l'écriture de l'utopie depuis la Renaissance prend en effet deux formes. La première ressemble davantage à un traité ${ }^{11}$, et la deuxième adopte des tours plus romanesques. Si on peut concevoir de commencer un roman par une explication théorique des fonctionnements d'une société idéale, le long roman galant français privilégie davantage les incipit in medias res, avec un moment de crise qui annonce un grand déchirement (Lallemand, 2013). Chez Urfé, ce n'est pas simplement le couple principal qui parait imploser : bien plus important encore, quoique moins visible, c'est l'univers utopique de ces bergers qui tend alors à s'effondrer.

Ces fissures qui se dessinent dans cette zone de friction traduisent tout le problème que pose l'idée même d'utopie. Galathée est attirée par cet idéal de perfection que le berger représente. Pour Urfé, l'utopie est faible dans le sens où elle ne peut être confrontée directement à la réalité sans être victime de sa propre perfection. La crise qui secoue l'utopie pastorale de L'Astrée est directement liée à un problème de lieu : les deux univers, celui des bergers et celui des chevaliers, se sont l'un l'autre entrechoqués parce qu'un berger a pris involontairement la place d'un chevalier. Céladon s'est retrouvé à la place de Polemas. C'est donc un problème de lieu qui conduit l'utopie pastorale à la crise : parce que son représentant le plus parfait, Céladon, s'est trouvé rejeté dans la zone de friction avec l'univers chevaleresque, il y a eu échange entre deux personnages. Urfé se sert de cette confusion pour mettre en scène la fin d'un monde utopique. L'arrivée inopinée de Céladon dans cet univers plus réaliste et qui n'est pas le sien provoque ensuite de nombreuses rencontres avec l'utopie, puisque avec Léonide, c'est aussi Pâris, fils d'Adamas et son cousin, qui commence à trouver un grand intérêt à tous ces bergers qu'il ne connaissait que de loin. Amoureux de Diane, il se fait luimême berger, délaissant alors le prestige que son rang lui conférait dans le monde d'Amasis. Si le monde des chevaliers semble éprouver un vif intérêt pour l'utopie pastorale, l'inverse n'est pas vrai. Cette rencontre ne profite donc pas à l'utopie, bien au contraire. On a vu que Céladon était bien marri de tant plaire à Galathée. Urfé propose le même schéma pour Diane et Pâris : si celui-ci est séduit par cette femme qui représente l'idéal au féminin, elle commence pour sa part à le trouver bien importun. L'utopie pastorale, par conséquent, ne souhaite ni dans un cas, ni dans l'autre se mêler à la réalité qui, bien loin de la mettre en valeur, la freine dans ses idéaux de perfection. Si Diane et Céladon ont des difficultés à discuter avec ceux qu'ils aiment, c'est à cause de l'intervention de ces personnages tirés d'un univers plus réaliste. La rencontre de ces deux mondes perturbe ainsi la dynamique utopique, et c'est ce processus qu'Urfé met principalement en scène dans le cadre de son récit principal, qui tourne majoritairement autour des couples Céladon/Astrée et Silvandre/ Diane. Même les plus parfaits des personnages chevaleresques et courtois ne peuvent

11. C'est le modèle que propose Thomas More : le livre II de L'Utopie est conçu comme un exposé très clair de toutes les caractéristiques de l'île ; rien de tel dans L'Astrée. 
par conséquent que nuire à l'utopie et ne s'en privent pas ${ }^{12}$ : ceci est un premier signe du pessimisme d'Honoré d'Urfé quant à l'idée même d'utopie. À quoi peut-elle bien servir si même les personnes de mérite ne peuvent prétendre à sa perfection?

\section{Pot de terre contre pot de fer : l'échec de l'utopie à soutenir les assauts de la réalité}

Ces grains de sable dans l'utopie que sont Galathée et Pâris ne font qu'annoncer un processus de destruction déjà bien visible. À force de se mêler des affaires du dehors, l'utopie pastorale court à sa perte. En l'identifiant dans un territoire qui n'est pas insulaire, Honoré d’Urfé la rend vulnérable.

\section{1. L'intrusion d'étrangers brutaux}

Un pont est possible avec l'extérieur, et il est facile d'entrer par méprise dans cette utopie. Dès lors, ce monde idyllique de la pastorale est menacé. Certes, quelques faits de violence avaient déjà existé avant que des étrangers ne viennent y porter le trouble, mais ils ne concernaient que des personnages de la génération précédente : Alcippe avait été en contact avec l'extérieur. Autrement, les faits de violence sont renvoyés à des temps immémoriaux, et à la naissance de la légende de la fontaine de vérité d'amour.

Le premier cas que l'on relève - l'agression de Diane par un chevalier étranger intervient rapidement dans la première partie roman. Il représente alors le barbare complet, puisque c'est le seul avec lequel un problème de langue se pose, dans un roman où autrement tout le monde se comprend : «pour estre estranger, il n'entendoit point son langage » (Urfé, 2011, livre VI, p. 399). Il représente l'étranger absolu dans ce qu'il a de plus dangereux pour un monde qui ne vit autrement que de perfection : il se jette sur Diane alors qu'elle sétait endormie près de l'eau. L'altercation qui s'ensuit conduit à la mort tragique du premier amant de celle-ci, Filandre : Pour la première fois, le monde réel attaque l'utopie et la massacre. Il s'agit d'un message d'avertissement d'autant plus fort que Filandre fait partie de ces personnages qui sont en fait des doubles de Céladon, le berger idéal. C’est indirectement lui qui est tué. Or, ce chevalier était arrivé par hasard sur ces terres, il n'y avait rien d'intentionnel dans ses actions. Le danger se trouve ainsi directement lié à ce choix que fait Urfé de placer son utopie dans un lieu accessible à tous. Univers fragile parce que trop parfait, l'utopie pastorale ne résiste pas au choc de cette rencontre.

12. Pour Jacques Ehrmann, l'univers pastoral est « un dédoublement de l'univers aristocratique »; si cela est vrai, les autres sociétés représentées sont bien plus réalistes dans leur reproduction des phénomènes sociaux de cet univers. La pastorale est un dédoublement rêvé. Voir Ehrmann (1963, p. 88-89). 


\section{2. Une violence contagieuse}

Le monde des bergers devient alors le lieu de violences terribles. La première partie montrait déjà un presque viol suivi d'un assassinat, ce qui permet de relativiser quelque peu l'idée que l'on va vers un assombrissement progressif de l'atmosphère, en tout cas pour les récits se rapportant à l'intrigue principale (Plazenet, 2003). Les signaux étaient là dès le début. Ce qui, cependant, devait rester une exception se reproduit dans la troisième partie, et avec plus d'éclat encore, puisque cette fois le meurtre a lieu au premier plan de la diégèse : se portant au secours de Damon, qui est assiégé par les chevaliers de Polemas, Tersandre se fait percer de coups. Cette fois, ce ne sont pas des personnages originaires du monde utopique qui sont directement concernés, puisque si Filandre était berger, Tersandre comme Damon sont étrangers au Lignon et tous deux chevaliers. Seulement, Tersandre portait bien au moment de sa mort les habits de berger : les hommes de Polemas ne pouvaient pas savoir qu'il n'était pas de la région. Ils croyaient donc frapper un honnête gardien de moutons. La crise naît ainsi non pas du fait d'un étranger qui se retrouve par hasard transporté dans le monde pastoral, puisque l'agression de Diane est sans lendemain, mais par les actions d'un des principaux chevaliers de la reine Amasis, Polemas.

Mais si la perte de l'utopie pastorale est décidée, il serait faux de la mettre entièrement sur le dos de ses voisins les chevaliers. Polemas est certes directement responsable de ce sang, mais l'élément déclencheur vient bien de l'extérieur du Forez. Urfé ne choisit pas n'importe quels personnages pour provoquer la chute de son utopie : ce sont précisément ceux issus de l'un des rares récits secondaires qui reprend les codes du genre des histoires tragiques ${ }^{13}$ qui en sont cause, à savoir Damon, Tersandre et Madonte. Ils apportent avec eux la violence. C’est Madonte la première qui fait part de ses craintes, et souhaite quitter un monde qu'elle ne juge plus sûr. Et c'est la vue de Damon qui irrite Polemas au point qu'il lance à sa poursuite ses hommes. Ainsi, l'irruption de l'histoire tragique, son inverse complet, achève de faire basculer l'utopie dans la violence et de la corrompre tout à fait, même si, sans cet incident, les signes de dégradation que nous avons relevés laissent bien présager une triste fin pour ces bergers. Le pot de fer a eu raison du pot de terre, qui pensait toujours pouvoir offrir un asile à tous ces malheureux opprimés par l'injustice des hommes.

L’Astrée, œuvre clef dans le développement du genre romanesque en France, nous fait ainsi le récit d'une impossible utopie. Le pessimisme de l'auteur ressort d'autant plus que c'est uniquement à cause du voisinage d'univers plus réalistes qu'elle s'écroule. À quoi sert d'écrire des utopies si celles-ci ne peuvent apporter aucun enseignement durable à l'humanité ? Le Forez est la région où Honoré d'Urfé a passé de tranquilles années, loin des ennuis de la cour. À travers la chute de son Forez

13. Delphine Denis, commentant le passage, fait le lien avec ce genre populaire quand parait L'Astrée. Voir Urfé (2016, note 91, p. 335). 
idyllique, il nous raconte en fait comment le repos est impossible, même dans le coin le plus reculé et le plus détaché des affaires de la cour de France. L'Astrée raconte l'histoire d'un paradis qui se perd.

\section{RÉFÉRENCES}

Bonansea, M. (2014). Le discours de la guerre dans la chanson de geste et le roman arthurien en prose. [Thèse de doctorat préparée sous la direction de MM. Vallecalle, J.-C. et Valette, J.-R. Université Lumière-Lyon 2].

Bury, E. (2007). Les deux cultures d'Honoré d'Urfé dans L'Astrée : entre idéologie nobiliaire et paideia humaniste. Dix-Septième siècle, 235(2), 315-323.

Chamard-Bergeron, J. (2013). L’Astrée et les intermittences de la mémoire. Dans I. Daunais, (dir.), La mémoire du roman (p. 61-73). Montréal : Presses de l'Université de Montréal.

Desrayaud, A. (2012). Le père dans le Code civil, un magistrat domestique. Napoleonica. La Revue, 14(2), 3-24.

Ehrmann, J. (1963). Un Paradis désespéré. L’amour et l'illusion dans L’Astrée. Paris : Yale University Press et PUF.

Elslande, J.-P. van. (2002). Roman pastoral et crise des valeurs dans la France du premier XVII siècle. Dix-Septième siècle, 215(2), 209-219.

Fragonard, M.-M. (2009). Des genres nouveaux pour les mondains. Dans A. Viala (dir.), Le Théâtre en France (p. 141-148). Paris : PUF.

Genette, G. (1966). Figures. Paris : Seuil.

Gouédo-Thomas, C. (1992). Les fontaines médiévales. Images et réalité. Mélanges de lécole française de Rome, 104-2, 507-517.

Lallemand, M.-G. (2013). Les Longs romans du XVII siècle. Paris : Classiques Garnier.

Matzat, W. (2002). Tradition et invention dans L'Astrée d'Honoré d'Urfé. Dix-Septième siècle, 215(2), 199-207.

Plazenet, L. (2003). Inopportunité de la mélancolie pastorale : inachèvement, édition et réception des œuvres contre logique romanesque. Études Épistémé, 3. http://journals.openedition.org/episteme/7056

Rosellini, M. (2015). L'entreprise critique de Sorel : une œuvre de 'novateur' ? Littératures classiques, 86(1), 187-214.

Urfé, Honoré d'. (2011) [1612]. L’Astrée, Première partie. D. Denis (dir.). Paris : Honoré Champion.

Urfé, Honoré d'. (2016) [1614]. L’Astrée, Deuxième partie. D. Denis (dir.). Paris : Honoré Champion.

Urfé, Honoré d'. (2015) [1619]. L'Astrée, Troisième partie. Grande-Bretagne : FB Éditions.

RÉSUMÉ : Alors qu'au siècle précédent fleurissent les textes montrant des sociétés utopiques, Urfé reprend ce thème qu'il adapte à un univers pastoral dans L'Astrée. Cependant, plutôt que de se contenter d'en démontrer la supériorité, il représente la fragilité qu'il y a dans tout projet utopique : dès lors qu'elle est incapable de sur- 
vivre longtemps aux assauts du réel, à quoi peut bien servir une utopie ? La société idéale qui nous était proposée au début du roman s'effrite peu à peu pour se briser sous nos yeux. Entre critique de lécriture utopique et regard paternel porté sur des personnages voués à souffrir de cette rencontre contre-nature avec l'extérieur, L'Astrée nous donne à voir comment le plus beau des pots de terre est inévitablement condamné à se confronter au pot de fer de la réalité. Le roman met alors en scène la beauté de cette brutale rencontre.

Mots-clés : utopie, pastorale, réalité, violence, destruction, Honoré d’Urfé

\section{The too weak utopia of L'Astrée: a variation on the earthen vs. iron pot theme}

When in the 16th century multiple texts present utopian societies, Urfé adapts this theme for a pastoral universe in L'Astrée. However, instead of proving its superiority, he demonstrates the inherent weakness of every utopian project: what could any utopia be good for if it is incapable of long withstanding the assaults of reality? The ideal society presented at the beginning of the novel grows weaker with every new chapter, and is finally destroyed. Between denunciation of utopia as a genre and a paternal consideration of the characters doomed to suffer as a result of meeting the outside world, L'Astrée shows how the most beautiful of the earthen pots inevitably crashes against the iron pot of reality. The novel depicts the beauty of this crash.

Keywords: utopia, pastoral literature, reality, violence, destruction, Honoré d'Urfé 